\title{
Management de la coopération dans les systèmes complexes
}

Gilles Le Cardinal, Jean-François Guyonnet et Bruno Pouzoullic

\section{(2) OpenEdition}

1 Journals

Édition électronique

URL : http://journals.openedition.org/communicationorganisation/1786

DOI : 10.4000/communicationorganisation. 1786

ISSN : $1775-3546$

Éditeur

Presses universitaires de Bordeaux

Édition imprimée

Date de publication : 1 mai 1995

ISSN : 1168-5549

Référence électronique

Gilles Le Cardinal, Jean-François Guyonnet et Bruno Pouzoullic, « Management de la coopération dans les systèmes complexes ", Communication et organisation [En ligne], 7| 1995, mis en ligne le 26 mars 2012, consulté le 30 avril 2019. URL : http://journals.openedition.org/

communicationorganisation/1786 ; DOI : 10.4000/communicationorganisation.1786

Ce document a été généré automatiquement le 30 avril 2019.

(c) Presses universitaires de Bordeaux 


\title{
Management de la coopération dans les systèmes complexes
}

\author{
Gilles Le Cardinal, Jean-François Guyonnet et Bruno Pouzoullic
}

1 Qui pourrait nier, aujourd'hui, qu'une entreprise, une administration, une association, un établissement scolaire, une famille soient un système complexe?

2 Il suffit, pour s'en convaincre, de se référer à une définition de la complexité inspirée par Jean-Louis Le Moigne qui pourrait être la suivante :

3 - un système complexe est un système qui ne peut être réduit à une représentation unique, et qui, pour chacune de ses différentes représentations, met en œuvre une logique différente.

4 C'est la confrontation de ces différentes représentations, élaborées par différentes catégories d'acteurs, avec leurs critères d'évaluation propres, qui donne au système son caractère complexe, ce qui les distingue des systèmes compliqués, qui peuvent mettre en œuvre de nombreuses parties avec une structure sophistiquée, mais dont on s'accorde sur une seule représentation et sur un seul ensemble de règles. Le double aspect corpusculaire et ondulatoire de la lumière en est un exemple dans le domaine de la physique, comme tout système qui allie l'homme et la technique, ou tout simplement enfin, un système constitué par deux acteurs qui participent à un même projet, ce qui nous assure, en l'occurrence, la présence d'au moins deux représentations et de deux modes de raisonnement distincts, autant que d'acteurs.

5 Dans une entreprise, les points de vue des actionnaires, du conseil d'administration, du directeur, des cadres, des employés, des clients sont bien différents! Dans un établissement scolaire, la logique de l'administration, du corps professoral, des parents et des élèves n'est à l'évidence pas la même, que dire de leur représentation respective du système et de leurs attentes.

6 L'efficacité et la fécondité du fonctionnement d'un système humain complexe est fondamentalement dépendant du degré de coopération qui s'instaure entre les parties qui le constitue. Ne pas s'en préoccuper, faire comme si l'instauration d'un climat de coopération était un «plus » facultatif, ou enfin, tenter de faire fonctionner le système 
malgré un climat conflictuel, sans s'y attaquer vraiment, sont des attitudes certes courantes mais à dépasser.

7 Nous proposons, au contraire, un nouveau style de management qui se fixe parmi ses priorités la «sûreté de la coopération » entre tous les membres du système, ce qui passe par la gestion raisonnée des confiances interpersonnelles.

8 Se donner un tel but ne relève-t-il pas d'une naïveté peu compatible avec le réalisme nécessaire à la conduite d'un système complexe?

9 Notre propos consiste d'abord à mieux définir cet objectif, à le clarifier, puis à proposer une méthodologie éprouvée dans l'industrie et dans le domaine social, susceptible d'accompagner le changement de climat souhaité. Aux responsables concernés de juger si notre méthode, intitulé PAT-Miroir, présente un intérêt pour eux.

\section{Description simple des systèmes complexes}

10 Pour clarifier le problème posé, nous proposons, tout d'abord, une façon simple de décrire un système complexe, tâche paradoxale, s'il en est, mais pédagogiquement utile pour pouvoir manipuler des concepts clairs et en tirer des conclusions opérationnelles.

11 Pour sortir du paradoxe, il suffit de donner une définition simple d'un système et de décrire le processus qui permet sa complexification progressive. On comprendra alors, par extrapolation, ce qu'est un système complexe.

12 Décomposons un système en trois entités :

13 - ses parties qui sont constituées des personnes, des machines et des processus qui y opèrent et de l'environnement dans lequel il s'insère.

14 - les interactions qui relient les parties entre elles que nous caractérisons par :

- des transferts d'information,

- des pilotages en commun d'action,

- des créations de relation,

- des découvertes d'identité respective.

15 - ses projets que l'on caractérisera par :

- les objectifs à atteindre,

- les contraintes à respecter,

- les critères à prendre en compte.

Un système simple, dans notre perspective, se caractérise par le fait qu'il ne comporte que peu de parties, par exemple, une seule personne ou qu'une catégorie de personnes, une ou plusieurs machines, que l'acteur ou la machine ne réalise qu'une seule tâche, ou un ensemble de tâches séquentiellement répétitives, que l'objectif est unique avec des contraintes précises et un critère claire à optimiser.

Dans ce cas la résolution de problèmes, la prise de décisions, les stratégies à mettre en œuvre, sont à définir par une seule personne. C'est, par exemple, le cas où un acteur seul est capable de réaliser la tâche. C'est aussi l'ancien mode de management où, s'il n'est pas capable de faire tout le travail seul, le directeur décide seul les objectifs, les stratégies, les procédures et les personnes qui les mettront en œuvre. Le personnel n'a qu'un rôle d'exécutant, dont il est simple de vérifier les performances réelles par rapport aux résultats attendus. Un système simple peut se complexifier de plusieurs façons : 
18 - en augmentant le nombre de ses parties, donc le nombre de participants, leur autonomie, la variété de leurs compétences, le nombre des méthodes à mettre en œuvre, des processus à piloter.

19 - en multipliant les interactions entre les parties ou en augmentant l'importance et la qualité de ses interactions :

20 - création de réseaux d'information,

21 - prise de décisions collectives, coordination des initiatives et des responsabilités, délégations,

22 - en renforçant la confiance entre les personnes, ce qui permet une plus grande prise de risques collective,

23 - en reconnaissant la spécificité de chaque acteur en diversifiant les compétences des acteurs et en acceptant de se révéler plus profondément aux autres,

24 - en incorporant de nouveaux projets, c'est-à-dire, en diversifiant les objectifs, en imposant de nouvelles contraintes, en donnant plus de critères à prendre en compte pour l'évaluation des résultats obtenus, multipliant les rationalités.

25 Tout ceci aura pour conséquence de multiplier les rationalités, les logiques et les stratégies possibles.

26 Rapprocher deux systèmes, qui ne travaillaient pas ensemble auparavant, nous assure de déboucher sur un système complexe puisque devront apprendre à cohabiter au moins deux représentations, deux structures, deux expériences, deux projets, deux logiques...

27 Prenons l'exemple de la complexification du système " entreprise » qui va s'opérer, si on se fixe comme nouveau critère : «l'amélioration de la coopération entre ses parties : la direction, le personnel administratif, le personnel d'encadrement, les agents de production et de maintenance, les commerciaux... »

28 On s'aperçoit, tout de suite, de l'incidences de l'introduction de ce critère qui va multiplier les contraintes, augmenter le nombre et la qualité des interactions nécessaires, modifier les objectifs existants et en introduire de nouveaux. Aussi serait-il prudent de fixer des priorités et de ne pas se disperser en imposant trop de changements au système en une seule fois.

29 On pourra, suivant les cas et les situations locales, se focaliser sur les relations entre « la direction et les cadres » ou « la production et la maintenance » ou bien « la production et le service commercial ", autant de combinaisons 2 à 2,3 à $3, \mathrm{n}$ à n possibles autant de projets distincts. La première étape consiste à préciser ensemble le projet particulier qui va cristalliser les réflexions des acteurs qui y sont engagés.

\section{La sureté de la coopération}

Améliorer la coopération entre certains acteurs ou groupe d'acteurs est une idée bien vague qu'il convient de préciser et d'expliciter. Prenant appui sur la théorie de la sûreté des systèmes techniques, nous nous proposons d'étendre les concepts de fiabilité, de maintenabilité, de disponibilité et de sécurité définis pour les systèmes techniques à la coopération interpersonnelle elle-même.

31 Partons de l'idée que chaque acteur impliqué dans un projet a le choix de coopérer ou de ne pas coopérer à chaque instant, à chaque étape, en chaque lieu, avec chacun des 
participants. La coopération est donc un processus instable qui risque à chaque instant de se détériorer en non-coopération, en compétition voire en conflit ouvert.

Nous déduisons de ces prémisses les définitions suivantes :

\section{Fiabilité de la coopération :}

«Capacité de compter sur la coopération de tous, nécessaire à la réussite pendant toute la durée du projet".

34 Il s'agit de rester, sans jamais en sortir, dans l'issue de coopération mutuelle des dilemmes successifs rencontrés pendant toute la durée du projet. Compte tenu du fait que des impondérables et des aléas peuvent se produire, la fiabilité de la coopération devra se fonder sur un projet très fructueux, et/ou sur des prises de garanties importantes, et/ou sur une confiance proportionnée aux risques que l'on estime pouvoir rencontrer lors du déroulement du projet.

\section{Maintenabilité de la coopération :}

35 «Capacité de rétablir la coopération interrompue avant le temps où elle sera de nouveau indispensable à la réussite du projet ».

On souhaite ici être capable de revenir à l'issue de coopération mutuelle, soit après la trahison d'un acteur, soit après l'issue de non-coopération mutuelle, soit après l'interruption du projet: le problème est alors de résoudre un conflit et de réparer ses conséquences ou de pouvoir redémarrer de façon coopérative un projet arrêté.

\section{Disponibilité de la coopération :}

37 "Capacité d'obtenir la coopération de tous à une étape quelconque du projet "

38 Cette qualité consiste donc à être assurée de se trouver, à une étape donnée, dans l'issue de coopération mutuelle, indépendamment des événements du passé.

39 Ce qui compte ici c'est d'obtenir le concours de tous à un moment jugé important. C'est lié à la capacité de remobilisation et réunification d'une équipe qui a pu se démotiver, se disperser ou s'agresser dans le passé.

\section{Sécurité de la coopération :}

40 «Capacité d'éviter l'apparition de rupture de coopération aux conséquences catastrophique pour le projet ou pour les acteurs. "

41 L'important est ici que l'on puisse compter sur la coopération de tous aux moments dangereux du projet, moment où on risque l'échec du projet et donc de tout perdre.

42 Améliorer la fiabilité de la coopération revient à augmenter la confiance des acteurs dans le système, dans les autres acteurs et dans le projet commun.

43 Améliorer la fiabilité de la coopération, c'est d'abord être capable de l'évaluer et de la comparer aux risques inhérents au projet.

44 Savoir assurer «la maintenance de la coopération », c'est se doter d'une méthode de résolution de conflit acceptée de tous et dont on connaît l'opérationnalité. 


\section{Les conditions d'application de la méthode PAT- Miroir}

$$
\text { objectif : }
$$

55

- L'approche doit être systémique et prendre en compte, tout particulièrement, les interactions entre les différents acteurs. Elle doit contribuer à former les acteurs concernés aux concepts de la systémique et à leurs mises en œuvre concrètes sur le système auquel ils participent. C'est une première phase indispensable que de décrire, précisément en groupe, les parties, la nature des interactions et le projet de système. En effet, la méthode n'a pas de sens en elle-même mais doit être utilisée pour faciliter la réalisation d'un projet qu'il convient de définir précisément en groupe. Nous dirons que cette phase permet d'établir un fonds commun d'évidences nécessaire à une bonne communication au sein du groupe et une bonne compréhension des concepts associés à la démarche proposée. avoir en l'autre acteur. S'il y a un risque de non-coopération de la part de l'autre, ne vautil pas mieux se prémunir en ne s'engageant pas dans la relation ou en refusant de coopérer soi-même? Toute méthode, sous peine d'être rejetée pour cause de naïveté, doit donc prendre en compte le fait que tous les acteurs sont libres de coopérer ou pas. Comme personne n'est sans défaillance, même menée avec une volonté de coopération, la mise en acte peut être défaillante et donc être interprétée à tort comme non-coopérative. 
60 Une façon de prendre en compte ces réalités incontournables consiste à prendre conscience que toute interaction génère simultanément trois sentiments dont l'intensité dépend de la situation et de l'acteur considéré :

61 - une " peur » de la non-coopération de l'autre acteur, de sa trahison, de l'exploitation qu'il peut faire d'une attitude coopérative unilatérale,

62 - un «attrait » pour la coopération mutuelle qui permettrait d'atteindre les résultats positifs d'une interaction réussie: meilleure information des acteurs, meilleure coordination dans l'action, meilleure confiance interpersonnelle, meilleure image de soi, des autres et du système.

63 - une «tentation » d'utiliser la coopération de l'autre acteur à son propre profit, voire de trahir sa confiance pour l'exploiter, récolter, à son seul profit, le bénéfice de l'effort de l'autre acteur, ou même une tentation de tirer partie de la situation pour blesser l'autre, l'attaquer, accroître le pouvoir qu'on a sur lui.

64 Peurs, attraits, tentations cohabitent dans le cœur des acteurs chaque fois qu'ils s'engagent dans un projet interactif. C'est un dilemme permanent dont on retrouve la structure dans le dilemme du prisonnier ${ }^{1}$.

65 Si les enjeux de l'interaction sont forts, seule la confiance en l'autre acteur accumulée dans le passé peut permettre de s'engager dans une attitude coopérative. La méfiance, au contraire, garantie une attitude défensive, non coopérative ou même agressive.

66 Si ces sentiments cohabitent au sein de chaque acteur, le premier pas consiste à en prendre conscience par une mise en mots.

67 Comment y parvenir simplement?

68 En proposant un atelier de créativité collectif et structuré de la façon suivante :

69 On commence par construire un tableau à double entrée où l'on porte en ligne et en colonne les différentes parties du système étudié. Chaque case du tableau correspond à l'interaction de la partie I, par exemple le service production, avec la partie j, par exemple le service maintenance. Pour décrire cette interaction avec précision, on demande à l'ensemble du groupe de se mettre à la place de la partie I (le service production) et d'exprimer ce que cette partie ressent vis-à-vis de la partie $\mathrm{j}$ (le service maintenance). Les participants doivent donc faire un effort de décentration en essayant d'imaginer les peurs, attraits et tentations que la partie I, le service production ressent vis-à-vis de la partie $\mathrm{j}$, le service maintenance. Les membres du service production participent comme les autres à cet effort de mise en mots structurée.

70 Un processus de complexification du regard de chacun sur les interactions du système s'opère progressivement. Les visions individuelles s'enrichissent mutuellement, on prend l'habitude de quitter son propre point de vue et d'essayer de se mettre à la place de l'autre.

71 Ce travail, facilité par sa réalisation en groupe avec un animateur compétent, est source d'une meilleure compréhension des problèmes que peut rencontrer la coopération au sein du groupe-projet. Chacun peut ainsi mieux identifier les risques comparés des différentes interactions du système.

72 Ne fait-on pas de même avant de se lancer dans une course en montagne lorsqu'on fait un inventaire des passages difficiles et que l'on détaille la nature des dangers que l'on risque de rencontrer, pour mieux s'équiper, s'entraîner et se rendre apte, à l'avance, à y faire 
face. « Mieux vaut se savoir en danger que se croire en sécurité » est la maxime de l'étude de la sûreté des systèmes que nous faisons nôtre ici.

\section{Le cœur de la méthode}

Dire ses peurs, c'est décrire, en fait, une vision négative du système, donner une représentation verbale des échecs possibles du projet, exprimer les comportements que l'on craint de voir apparaître. Mais tout le monde a ses propres peurs. Les exprimer en commun présente l'avantage de construire une représentation collective de l'image négative du système. On est rarement autorisé à faire ce type de travail, généralement considéré comme négatif et potentiellement destructeur, ce qui est possible si on s'y arrête. Pour que tout le monde participe, la meilleure façon de procéder consiste à réaliser un atelier de créativité structuré où le groupe est appelé à se mettre à la place d'un des participants et d'exprimer ses peurs. Plus on craint l'échec, plus on est productif, curieux paradoxe où, la source de blocage, devient source d'informations ouvrant la possibilité de les surmonter.

74 A contrario, dire ses attraits, c'est décrire une vision positive du système, donner une représentation verbale des succès possibles du projet, décrire les comportements que l'on souhaite voir apparaitre, exprimer ces attentes les plus profondes. Cela devient maintenant possible puisque les peurs ont été prises en compte, même les opposants les plus farouches peuvent être mobilisés pour cette tache. En procédant de même sous forme d'atelier de créativité structuré, on élabore ainsi une vision collective de l'image positive du système.

75 Dire ses tentations, c'est beaucoup plus difficile! En effet, on préfère en général ne pas voir les tentations auxquelles on est soumis. Le problème vient de ce que mes tentations sont précisément l'une des sources des peurs de ceux qui travaillent avec moi :

76 - autoritarisme, décision prise seule, mauvais partage du travail, rétribution insuffisante des services rendus, reproches incessants,...

77 Il est bien plus facile, par conséquent, d'expliciter les tentations des autres que les siennes propres. C'est le service que va rendre, précisément, un travail de groupe qui a cet objectif. Si besoin est, on pourra d'ailleurs répartir des peurs exprimées par les autres pour reconstruire certaines tentations des uns.

78 Le deuxième intérêt de ce travail est de commencer à établir une relation de cause à effet entre l'image négative du système et ce qui peut faire que cette image devienne réalité. On a peur, en effet, que l'autre cède aux multiples tentations qui l'assaillent sans même, parfois, qu'il en soit conscient.

79 Si on sait à l'avance que le système peut basculer, à tous moments, dans la noncoopération, on saisit mieux, après ce travail par quels types de mécanismes cela risque d'arriver.

80 Mais l'avantage du procédé qui consiste à faire l'inventaire des tentations possibles des différents acteurs réside surtout en ce qu'il se distingue de la recherche du coupable. Dire qu'une tentation existe ne signifie ni que l'on y ait déjà cédé, ni que l'on va y céder à l'avenir. C'est une sorte de balisages des dangers possibles dont le groupe devient collectivement plus conscient. 


\section{Construire une représentation collective}

81 Cette construction collective d'une représentation plus complexe que chacune des représentations individuelles du système n'est pas le moindre des avantages d'une telle façon de procéder.

Toutefois, la présence d'un animateur formé à la méthode et extérieur au groupe humain concerné est absolument nécessaire pour éviter les risques de dérapages qui constituent la tendance de certains à la chasse aux sorcières et à l'ouverture de placards où gisent parfois de funestes cadavres.

Le dernier avantage de la démarche proposée, consiste à l'élaboration d'un nouveau fonds commun d'évidences entre les participants qui soit très lié au projet. Ce n'est pas un mince résultat que d'avoir réussi à écouter l'autre, à respecter son idée au même titre qu'il a écouté et respecté la sienne. Toutes les idées seront notées côte à côte et auront a priori le même poids. Au passage, un deuxième apprentissage s'est accompli : réussir à mettre des mots sur des ressentis qui sont bien rarement exprimés, cela contribue à clarifier les rapports humains et de réduire les non-dits si souvent source de bien des malentendus.

Cette expérience forte est de nature à faire toucher du doigt à chaque participant le problème philosophique « du même et de l'autre ». Je suis capable de comprendre ce que l'autre exprime mais je n'ai pas le même ressenti. Les différences d'expériences, de caractères, de valeurs, de croyances sont explicitées sans craindre le conflit ou l'exclusion, de par la double protection et de la méthode et de l'animateur.

Sur le plan psychologique, se mettre à la place de l'autre en pensée, évitera de prendre la place de l'autre en acte, ce qui est souvent source de conflit voir de violence. L'expérience humaine que constitue le temps passé au travail en groupe n'est pas négligeable, d'autant qu'au cours de la séance l'anodin côtoie le dramatique, les temps où l'on est personnellement très concerné alternent avec ceux où l'on est plus observateur.

Sous l'influence de l'animateur, voire par son action énergique parfois nécessaire, personne n'est accusé, culpabilisé, pris comme bouc-émissaire. Toutefois chacun peut faire son miel de l'information qui s'exprime C'est une chance donnée à chacun de découvrir le système au sein duquel il vit et travaille sous un jour nouveau pour lui mais en rien normatif. Comme la trace du travail du groupe est distribuée, chaque participant pourra en profiter individuellement pour l'étudier à loisir, s'il le souhaite.

Si la phase de mise à plat des peurs, attraits et tentations, présente, comme nous l'avons vu, un réel intérêt en soi, et constitue une condition nécessaire à l'établissement d'une meilleure coopération, elle ne saurait être, à elle seule, capable d'instaurer un changement de climat réel et durable.

Elle doit être complétée par une seconde phase plus proche de la prise de décision et d'engagement dans l'action.

\section{L'évaluation individuelle anonyme}

Avant d'envisager cette deuxième partie, le travail collectif s'interrompt de manière à permettre à chacun d'évaluer personnellement, mais de façon anonyme, la totalité des peurs, attraits, tentations exprimés. Il s'agit de donner un poids à l'importance des 
risques que constituerait, pour la coopération, la réalisation des événements redoutés (peurs et tentations) et à l'importance des potentialités de gains contenues dans la réalisation des événements souhaités (attraits). Tous les avis sont ainsi pris en compte, sous forme de notes, de façon démocratique et égalitaire, l'anonymat garantissant la possibilité réelle de donner un point de vue personnel voire partial sans représailles possibles.

Par le calcul, les notes sont intégrées et les résultats du groupe visualisés par items. Les peurs, attraits, tentations sont alors classés dans l'ordre d'importance décroissant donné par le groupe. C'est ce document qui constituera la base de la seconde partie du travail où on va construire une représentation collective du projet, un ensemble de préconisations et d'un tableau de bord de la coopération dans le groupe.

\section{Constructions des préconisations du groupe}

91 Parti d'une mise en commun du ressenti psychologique individuel, le travail va se transposer maintenant sur le plan cognitif où l'on va faire appel à l'intelligence des participants pour un travail d'organisation et de classement systématique. Le premier objectif de cette phase consiste à rassembler les peurs, attraits, tentations en différents sous-ensembles.

On constitue ainsi des paquets de peurs, d'attraits, de tentations qui se rapportent tous à un même thème. Le jeu consiste alors pour le groupe à donner un nom à chacun des thèmes que l'on qualifiera de thèmes incontournables. C'est une façon collective de faire l'inventaire de tous les facteurs qui jouent un rôle majeur dans la réussite du projet. On a réalisé ainsi la décomposition du projet en différents sous-projets clairement identifiés et détaillés par la liste des items associés.

93 Ces thèmes incontournables constituent une nouvelle représentation du système, construite logiquement par le groupe, intégrant équitablement tous les points de vue individuels.

94 D'un point de vue cognitif, comme d'un point de vue sociologique, cette procédure de construction est très intéressante. Bien sûr, la liste des thèmes incontournables n'est pas unique et dépend largement du groupe qui l'a élaboré.

Certes, on a pu expérimenter le fait que deux groupes partant de la même liste ordonnée des items, n'aboutissait pas exactement au même résultat :

96 - l'un trouvait treize thèmes, l'autre onze thèmes dont sept étaient communs ou possédaient des titres extrêmement voisins

97 - certains thèmes étaient dédoublés dans un groupe, un autre au contraire plus vaste en joignait trois en un seul

98 - un thème avait été totalement omis par l'un des groupes.

99 Ces différences mettent en évidence le fait que la construction des thèmes incontournables constitue le résultat du travail d'un groupe bien précis.

100 Si les groupes travaillent de façon totalement indépendante sans mettre en commun la liste des items, les résultats au niveau des thèmes incontournables peuvent alors être complètement différents. Il est alors très intéressant et tout à fait significatif de comprendre l'origine des différentes représentations obtenues compte tenu des caractéristiques des participants de chaque groupe. 

commun. répondrons-nous.

- L'élaboration d'un ensemble de préconisations classées par thèmes incontournables visant à faire baisser les peurs exprimées, à limiter si possible les tentations, à renforcer et à faire passer dans les faits les attraits potentiels.

Ces préconisations seront élaborées là encore sous forme d'un atelier de créativité ciblé.

- L'élaboration d'un ou plusieurs critères permettant d'évaluer la qualité atteinte relativement à chaque thème. Ces critères serviront à la constitution d'un tableau de bord permettant de suivre les progrès de la coopération dans le groupe-projet.

Ainsi le travail réalisé débouche sur une sorte de charte de comportements souhaités relatif à un projet précis, associée à un tableau de bord permettant de juger de l'état de la coopération au sein du projet sur un référentiel bien défini et accepté par tous.

Cela ouvre la possibilité de réévaluer périodiquement, et la pertinence de la charte et les progrès du groupe lors de réunions ultérieures où l'on demandera à chacun, anonymement, d'évaluer l'état des relations du groupe sur le référentiel élaboré en

Le parcours proposé nécessite que le groupe prenne du temps et investisse dans une telle démarche, temps perdu diront certains avides de résultats rapides et spectaculaires, temps nécessaire pour apprendre à travailler ensemble avec patience et méthode

\section{Réflexions sur la méthode}

\section{La métaphore du Carrefour européen}

8 La célèbre métaphore du "carrefour européen » introduit par De Mailly et repris par Jean-Louis Le Moigne ${ }^{2}$ nous permettra de mieux justifier l'investissement que constitue la mise en œuvre de la méthode. Lorsque deux routes se croisent, on peut régulariser le trafic de façon autoritaire et externe au système par des feux tricolores. Alors, bien souvent, on vit péniblement la situation stupide où un feu rouge nous arrête alors que personne ne vient sur l'autre voie. Il faut alors obéir, même si l'ordre n'est pas adapté à la situation, puisque c'est l'obéissance aveugle et bête de tous qui rend la solution malgré tout viable, quoique peu performante.

9 La solution nouvelle apportée par le carrefour européen est paradoxale. On construit un obstacle au centre du carrefour. Tout le monde est obligé de ralentir mais alors, les risques d'accidents les plus graves étant évités, on fait appel à l'intelligence de chaque conducteur, à sa faculté d'adapter son allure à la situation observée, tout en respectant une règle simple et évidente : " priorité à l'évacuation du carrefour ".

Chacun est responsabilisé, possède une partie de la décision et doit faire confiance aux autres dans leur capacité de prendre les bonnes décisions en fonction de la situation.

1 C'est ainsi que fonctionne notre démarche, non plus lors d'une intersection, mais lors d'interactions humaines complexes. Elle joue le rôle de l'obstacle qui oblige à ralentir. On ne va pas se lancer sans précaution dans le projet, mais on va lever la tête et prendre conscience de tous les risques potentiels. Le groupe est appelé à mieux percevoir la situation, à construire une représentation plus complète, plus complexe. 
112 Alors, et alors seulement, chacun ainsi responsabilisé et informé, pourra s'engager en connaissance de cause dans le projet en étant conscient des sous-projets, des règles à respecter pour le bien de tous et des indicateurs de qualité.

\section{Les conditions d'application de la méthode}

Comment prendre du recul par rapport à la démarche proposée, déterminer ses limites, souligner ses points forts et ses points faibles?

114 La première des conditions de son application est l'accord de principe du groupe sur l'emploi de la méthode après en avoir pris connaissance. Toutes les étapes doivent lui être clairement décrites. Même si les résultats du travail ne sont pas connus d'avance, puisque la méthode n'est qu'un cadre vide à remplir par les participants eux-mêmes, on connaît précisément chaque étape du chemin à parcourir.

115 La sincérité de la démarche, c'est-à-dire l'assurance qu'il ne s'agit pas d'une manipulation de la direction, est une condition essentielle au succès de l'application de la méthode. Cette condition fait partie des règles déontologiques à respecter par l'animateur qui doit exiger dans le contrat qui le lie au demandeur que soit inscrite une clause spécifiant qu'aucune solution préalable n'est attendue, suggérée, ou privilégiée comme résultat du travail. C'est bien au groupe d'élaborer sa charte et son tableau de bord et non à l'animateur de le conduire dans telle ou telle direction prédéterminée. Cela justifie également l'exigence que l'animateur ne fasse pas partie du système humain en question.

La deuxième règle porte sur la constitution du groupe :

117 - Il est préférable que toutes les parties concernées soient représentées ; il est souhaitable que personne n'occupe une situation hiérarchique supérieure à toutes les autres. Ceci ne signifie pas que le directeur de l'établissement ne puisse pas participer au travail du groupe, mais on doit alors prévoir des ingénus, extérieurs au système et à sa hiérarchie qui pourront librement exprimer ce qui saute aux yeux mais que personne ne se sentirait autorisé à dire en présence d'un supérieur.

\section{Quelques raisons expliquant l'intérêt constaté de la méthode}

$118 \mathrm{Au}$ point de vue méthodologique, il faut bien comprendre que la première phase du travail consiste à décomposer le système initial en mille petits morceaux disparates, les fameux items de peurs, attraits, tentations. L'égalité des contributions est strictement respectée grâce à l'animateur dont c'est l'un des rôles principaux. Il peut même tempérer ceux qui auraient tendance à mobiliser la parole et cela suffit, en général, pour que le groupe se régule alors de lui-même. Une réaction en chaîne se déclenche, les pensées des uns suscitent des idées chez les autres. Le principe veut que toute peur exprimée soit prise en compte. On opère en ajoutant les items, jamais en en supprimant. Si le groupe dans sa majorité n'est pas d'accord, cela apparaîtra à la phase suivante, où l'item correspondant n'obtiendra qu'une très faible moyenne. À ce niveau personne ne peut bloquer le système. Ceux qui luttent pour qu'une peur énoncée soit supprimée s'aperçoivent vite qu'ils font fausse route.

119 Cette première partie ne faisant que peu appel à l'intelligence mais au ressenti, elle évite le piège des discussions où il s'agirait d'avoir raison sur l'autre. Si quelqu'un ressent une peur ou la juge possible, personne ne peut le contester. L'animateur est là pour veiller à 
arrêter un tel débat s'il apparaissait. Cette façon de faire évite la confrontation des représentations intellectuelles du système qui ne feraient que s'entrechoquer sans s'enrichir. La dislocation du système interdit à quiconque d'imposer un seul point de vue, une seule représentation qui serait obligatoirement réductrice. La technique des ateliers de créativité au contraire accentue la déstructuration du système et contribue à créer un climat de détente. Le rire est souvent au rendez-vous lors de l'expression de certains items. Bien des baudruches, artificiellement gonflées, sont ainsi démystifiées, ce qui contribue à détendre considérablement l'atmosphère et à construire un climat de travail confiant.

L'exercice qui consiste à se mettre à la place de l'autre est parfois une révélation chez certain qui n'avait jamais pris autant de temps pour le faire. D'autres sont particulièrement touchés que le groupe fasse l'effort de se mettre, en pensée, à leur place et d'essayer de comprendre leur problème. Ils ont enfin l'impression d'être écouté et cela de façon approfondie. La valorisation personnelle qui en résulte n'est pas un effet secondaire, mais peut être le déclenchement d'un changement d'attitude par rapport au groupe et au projet. On constate alors que la session est une source nouvelle de motivation.

121 La méthode agit dans cette phase comme une loupe grossissante qui permet de regarder les interactions une à une, ce qui la distingue nettement de toutes les techniques qui consistent à identifier la psychologie profonde des acteurs en présence ; on sait trop bien les peurs et les réticences qui résultent de telle démarche effectuée collectivement.

Après avoir examiné de très près les interactions, la méthode consiste à prendre alors un recul considérable, opérer un zoom arrière pour voir à nouveau le système dans sa globalité grâce à l'émergence de l'ensemble des thèmes incontournables. Mais, chose curieuse et intéressante, on ne voit plus du tout le système comme avant, le petit point de vue qui était le sien initialement s'est considérablement transformé et amplifié. On découvre qu'on possède maintenant une vision élargie, plus complète, et en plus, mieux partagée par le groupe. Bien sûr, chacun garde toujours sa perspective propre, sa position particulière et spécifique dans le groupe mais sa perception du projet est plus globale. Ses problèmes particuliers s'intègrent dans un tout plus vaste dont on perçoit mieux les contours et les interactions. Il ne peut plus défendre seulement son point de vue, ses contraintes, ses objectifs, ses critères car on a pris conscience au moins partiellement des autres points de vue, contraintes, objectifs et critères. Et les découvertes ne sont pas moindres chez le directeur que chez la secrétaire ou le professeur. Comme dans un hologramme, chaque membre du groupe est capable de donner une description globale du projet.

D'autre part, si les acteurs ont perdu de leur naïveté première, ils ont acquis une lucidité nouvelle à partir de laquelle ils peuvent, en toute connaissance de cause, faire le pari de la coopération en espérant qu'elle s'établira durablement entre les membres du groupe malgré les difficultés existantes, ou plutôt parce que, maintenant, l'ensemble les difficultés a été répertorié. La confiance devient alors une naïveté seconde, comme la définit Jacques De Bourbon Busset ${ }^{3}$, où l'acteur, lucide sur les trahisons possibles, fait le pari de confiance car il sait qu'on est payé de retour si les conditions sont réunies. Et le vécu du travail commun est de nature à donner cette assurance.

Après ces considérations issues de notre expérience et de celles de professionnels de la communication qui ont expérimenté et amélioré la méthode, il reste à donner des 
exemples d'application, ils sont nombreux, que ce soit dans le domaine social, en entreprise ou dans l'administration.

\section{Exemples d'application}

\section{Dans le domaine social}

125 - Apprendre à mieux déléguer dans un Foyer Socio-Educatif,

126 - Résoudre les conflits entre éducateurs et parents d'enfants handicapés dans un centre d'hébergement,

127 - Régler un différent entre un Conseil d'Administration et un Centre d'Aide par le Travail,

128 - Améliorer les comportements d'éducateurs dans une relation d'aide,

129 - Établir un guide d'entretien pour harmoniser les rapports entre l'agent des ASSEDIC et les demandeurs d'emploi,

130 - Faciliter le dialogue d'un enfant avec l'administration sociale et juridique.

\section{Dans le domaine de l'entreprise}

131 - Créer un climat pour favoriser la formation continue des vendeurs dans une entreprise commerciale,

132 - Régler un contentieux entre un directeur et un consultant,

133 - Améliorer les comportements d'écoute mutuelle entre partenaires professionnels,

134 - Définir le cahier des charges d'un nouveau produit mettant en jeu de multiples compétences et intervenants,

135 - Améliorer les relations entre un service maintenance et un service production,

136 - Faciliter le passage du stade «entretien » à celui de "maintenance préventive » dans une PME,

137 - Favoriser l'usage d'un nouvel outil informatique pour une catégorie de personnel inexpérimentée et hostile,

138 - Favoriser la coopération d'un expert à la construction par un cogniticien d'un système à base de connaissances.

\section{Dans le domaine administratif et scolaire}

139 - Favoriser la coopération inter-service pour préparer la création d'un guichet unique dans une administration départementale,

140 - Définir une stratégie d'école face à l'exclusion d'un enfant par ses camarades de classe,

141 - Mettre en place un projet pédagogique visant à favoriser la prise de confiance en soi des élèves... 


\section{Conclusion}

142 La variété de ces exemples ne doit pas faire croire que la méthode proposée est une panacée universelle, les limites déjà repérées de la méthode sont nombreuses :

143 - il faut disposer de temps en équipe pour aller jusqu'au bout de la méthode. S'arrêter en cours de route laisse un fort goût de frustration. Il faut compter une demi-journée pour la présentation de la méthode, trois fois quinze minutes par interaction pour la production des peurs, attraits, tentations. Pour un système comportant deux acteurs, l'étude des quatre interactions prend trois heures, pour trois acteurs ou groupes d'acteurs il faut une journée; bien sûr on peut regrouper les acteurs en classe d'acteurs aux enjeux voisins pour minimiser le nombre d'interactions à étudier. De même, pour les grands systèmes, on ne s'attardera que sur les interactions principales en négligeant celle dont l'importance est jugée moindre (à définir en équipe). La plus grande matrice des interactions rencontrées à ce jour comportait onze lignes et onze colonnes, soit 121 interactions dont une cinquantaine ont pu être étudiées en détail en cinq jours, par un groupe de douze personnes!

144 Bien entendu ce cas limite se justifiait par l'ampleur du projet étudié, sa réelle difficulté, et l'importance des enjeux.

145 - La méthode peut être utilisée en cas de conflit mais le conflit, s'il existe, ne doit pas être ouvert depuis trop longtemps et ne pas être focalisé sur deux personnes. Ce type de problème est mieux pris en compte par une thérapie de type psychologique que par notre approche systémique.

146 - Les participants doivent être impliqués dans le système, le pratiquer depuis un certain temps, le problème à résoudre doit être ressenti par eux comme pertinent. Il est difficile de faire travailler un groupe sur un problème futur qui ne les touche pas encore et sur lequel ils n'ont pas d'informations suffisantes.

147 - Le demandeur de l'intervention doit jouer le jeu et ne pas mettre de conditions sur les résultats obtenus. Il peut par contre préciser qu'il se réserve le droit de ne mettre en œuvre que partiellement les préconisations du groupe après un examen attentif et en justifiant les choix ainsi opérés.

148 - L'animateur doit être formé et compétent, sa personnalité intervient largement dans le climat de travail et la qualité des résultats obtenus. Il doit pouvoir répondre à toutes les objections soulevées à propos de la méthode et éviter les inévitables dérives possibles.

149 - Enfin, il faut être conscient du fait que la méthode, de par sa puissance même, peut devenir dangereuse. En effet, elle peut être détournée de son but et utilisée de façon perverse pour alimenter un conflit. Elle peut se révéler alors d'une efficacité redoutable pour celui qui voudrait attiser les peurs, anéantir les attraits, exacerber les tentations.

150 Comme tout bon outil, la méthode doit être utilisée avec compétence et discernement, raison de plus pour en réserver l'usage à des professionnels compétents. Mais la meilleure façon de se faire une idée de la méthode est de participer à sa mise en œuvre. On constate en général que les participants à une session en sont les meilleurs porte-parole.

151 La méthode proposée, et c'était notre ambition de le démontrer, permet d'agir sur les quatre composantes de la sûreté de la coopération en permettant d'évaluer sa fiabilité, en servant de guide à la résolution de conflits éventuels, en faisant des préconisations sur la 
marche à suivre pour réaliser le projet harmonieusement, de façon coopérative et en bonne sécurité.

\section{NOTES}

1. Le Cardinal, G., Guyonnet, J.F., «Les mathématiques de la confiance » Pour La Science, $n^{\circ} 81$, juillet 84, p 71-77.

2. Le Moigne, J-L. Confiance et complexité, Actes du colloque « Du mépris à la confiance », dec 90, UTC-IDTH, p 74-92.

3. De Bourbon Busset, J., La stratégie de la confiance. Actes du colloque - Du mépris à la confiance, dec 90,UTC-IDTH, p. 28-32.

\section{RÉSUMÉS}

Face à la nécessité d'obtenir une coopération fiable dans une équipe interdisciplinaire chargée d'un projet complexe, nous proposons une méthodologie originale s'appuyant sur la modélisation de la complexité, de la communication et de la sûreté des systèmes socio-tehcniques. La méthode PAT-Miroir propose à tous les participants un lieu de paroles restructurant, où ils sont conduits par un animateur à complexifier leur représentation personnelle du projet et à construire une représentation collective des interactions nécessaires à sa réussite. La démarche part des ressentis positifs et négatifs des différents acteurs, passe par une évaluation individuelle de l'importance des facteurs ainsi identifiés et aboutit à la construction d'une série de préconisations concrètes, assurant une coopération plus attractive et plus sûre.

To meet the increasing need for reliable and effective cooperation within interdisciplinary groups in charge of complex projects, we propose a new and original method. This method is based on modélisation of complexity, communication and dependability of sociote-chnical systems. This method called « PAT-Mirror», requires grouping all members of the project group and to guide them in a structured talk monitored by a tutor. To be successfull, members must describe (complexify) their personnel representation about the project and build a collective representation about the project and build a collective representation through their interactions. The approach starts by defining feelings and intuitions (either positive or negative) of different actors, followed by each actor's individual assesment of importance of the identified factors. The last step consists in making series of practical recommendations which allow more effective and reliable cooperation. 


\section{AUTEURS}

\section{GILLES LE CARDINAL}

Gilles Le Cardinal est professeur de communication à l'UTC. Sa thèse d'habilitation à diriger les recherches prote sur la modélisation systèmique de la communication interpersonnelle finalisée.

\section{JEAN-FRANÇOIS GUYONNET}

Jean-François Guyonet, Docteur d'Etat est spécialisé dans la sécurité des systèmes et travaille sur des problèmes de fiabilité technique et humaine.

\section{BRUNO POUZOULLIC}

Bruno Pouzoullic, consultant e entreprise, a rendu opératoire la méthode PAT-Miroir à travers sa mise en application dans de nombreuses entreprises et administrations. Il est chercheur associé à l'équipe IDTH. Ils coopèrent dans le cadre de l'équipe IDTH (Interactions de Défaillances Technologiques et Humaines), au sein de l'unité de recherche COSTECH (Connaissance, Organisation et systèmes Techniques) de l'UTC. 